Preprint HU-TFT-94-14

\title{
LARGE SCALE INHOMOGENEITIES FROM THE QCD PHASE TRANSITION
}

\author{
J. Ignatius $^{a}$, K. Kajantie ${ }^{b}$, H. Kurki-Suonio ${ }^{a}$ and M. Laine ${ }^{b}$ \\ ${ }^{a}$ Research Institute for Theoretical Physics and \\ ${ }^{b}$ Department of Theoretical Physics, \\ P.O. Box 9, FIN-00014 University of Helsinki, Finland
}

19 May 1994

\begin{abstract}
We examine the first-order cosmological QCD phase transition for a large class of parameter values, previously considered unlikely. We find that the hadron bubbles can nucleate at very large distance scales, they can grow as detonations as well as deflagrations, and that the phase transition may be completed without reheating to the critical temperature. For a subset of the parameter values studied, the inhomogeneities generated at the QCD phase transition might have a noticeable effect on nucleosynthesis.
\end{abstract}

PACS numbers: 98.80.Cq, 12.38.- -

Electronic addresses: ignatius@phcu.helsinki.fi, kajantie@phcu.helsinki.fi, hkurkisu@pcu.helsinki.fi, mlaine@phcu.helsinki.fi 


\section{Introduction}

Much of the interest in the cosmological first-order QCD phase transition has been due to the possibility of affecting the big-bang nucleosynthesis [1]. This would require creating significant inhomogeneities in the baryon number density, with a characteristic distance scale of the order of $10^{-3} t_{H}$. The typical distance $l_{n}$ between bubbles nucleated in the phase transition is related to the bubble surface tension, $l_{n} \propto \sigma^{3 / 2}$. The excitement has diminished as lattice calculations have yielded rather low estimates of $\sigma$, indicating much smaller distance scales.

The purpose of this paper is to point out that a low $\sigma$ does not necessarily imply short distances. The reason is that in addition to $\sigma$, the distance scale depends on the latent heat $L$. As the phase transition appears to be at most only weakly first order, surface tension and latent heat should both be small compared to energy scales at the transition. Using the bag equation of state and classical nucleation theory, we find that even for small $\sigma$, there is a large parameter space where the phase transition is preceded by considerable supercooling, and the distances between critical bubbles are large. Interestingly, we also find that with a more realistic equation of state than that of the bag model, the distances get even larger. It will be very enlightening to redo our calculations, when the whole equation of state of the QCD matter can be extracted from lattice calculations.

In view of nucleosynthesis, not only the distances between critical bubbles but also the later stages of the phase transition are important. Since we do not know the microscopic physics operating at the phase transition front, we cannot give a definite solution to the problem. However, we can analyze what processes are allowed by hydrodynamics. We find that the hadron bubbles could grow as detonations and that there is not necessarily a stage of slow growth in thermodynamical equilibrium during the phase transition. This leads to a rather unconventional picture of the phase transition. We also find that if this picture is correct, then it is unlikely that the QCD phase transition has a noticeable effect on nucleosynthesis, in spite of the large distance scales. On the other hand, it is also possible that the hadron bubbles grow as deflagrations, the distance scales are equally large as above, and the QCD phase transition may have a noticeable effect on nucleosynthesis. Clearly, for conclusive results, a better knowledge of the parameter values must be acquired.

The plan of the paper is the following. In Sec. 2 Nucleationsection.2 we review classical nucleation theory and calculate the nucleation temperature and the distances between critical bubbles in the QCD phase transition, using the bag equation of state. In Sec. 3The effect of the equation of statesection.3 we discuss the effects of a more realistic equation of state. Sec. 4Detonations and deflagrationssection.4 contains a hydrodynamical analysis of the possible growth mechanisms of the hadron bubbles, and in Sec. 5Baryon numbersection.5 we discuss the effect of the QCD phase transition 
on nucleosynthesis. The conclusions are in Sec. 6Conclusionssection.6.

Since the hydrodynamics of bubble growth in this context has sometimes been analyzed using a plane-symmetric geometry (i.e., $1+1$ dimensions), we stress that in this paper we use spherical geometry everywhere.

\section{Nucleation}

We review the classical thermal nucleation of thin-wall bubbles with the bag equation of state

$$
p_{q}=a_{q} T^{4}-\frac{L}{4}, \quad p_{h}=a_{h} T^{4},
$$

where $L=4\left(a_{q}-a_{h}\right) T_{c}^{4}$ is the latent heat. We use the notation $\hat{T} \equiv T / T_{c}$, where $T_{c}$ is the critical temperature of the phase transition. The radius of a critical bubble at temperature $\hat{T}<1$ is

$$
r_{c}=\frac{2 \sigma}{p_{h}-p_{q}}=\frac{8 \sigma}{L} \frac{1}{1-\hat{T}^{4}},
$$

where $\sigma$ is the bubble surface tension. The free energy expended to nucleate a critical bubble is

$$
W\left(r_{c}\right)=\frac{4 \pi}{3} \sigma r_{c}^{2}=\frac{16 \pi}{3} \frac{\sigma^{3}}{\left(p_{h}-p_{q}\right)^{2}} .
$$

The nucleation rate is

$$
p(t)=C T_{c}^{4} e^{-S(t)},
$$

where

$$
S(t) \equiv W\left(r_{c}\right) / T=\frac{256 \pi}{3} \frac{\sigma^{3}}{L^{2} T_{c}} \frac{1}{\hat{T}} \frac{1}{\left(1-\hat{T}^{4}\right)^{2}},
$$

and $C$ is a prefactor roughly of order unity.

There are thus two essential parameters, $L$ and $\sigma$. The latent heat $L$ gives the difference of $a_{q}$ and $a_{h}$. Otherwise the values of $a_{q}$ and $a_{h}$ affect the nucleation only slightly through the expansion rate of the Universe. We are interested in the case of a small $L$. Therefore, $a_{q}$ and $a_{h}$ cannot be taken to have their ideal gas values $51.25 \pi^{2} / 90$ and $17.25 \pi^{2} / 90$, which correspond to a large latent heat $L=14.9 T_{c}^{4}-$ but must lie closer to each other somewhere between these values. In reality they are functions of temperature $a_{q}(T), a_{h}(T)$, but until Sec. 3The effect of the equation of statesection.3, we take them to be constant.

With constant $a_{q}$ and small $L$, the expansion (cooling) timescale is approximated by

$$
t T^{2}=\text { const. }=t_{c} T_{c}^{2} .
$$

The Universe reaches the critical temperature at $t=t_{c}$. The Universe then supercools to $T<T_{c}$ and the nucleation rate begins to increase rapidly. A nucleated bubble 
grows by detonation or deflagration, preventing further nucleation inside the volume $(4 \pi / 3) v^{3}\left(t-t^{\prime}\right)^{3}$, where $t^{\prime}$ is the nucleation time. In the case of detonation the relevant velocity $v$ is that of the phase boundary (detonation front), $v=v_{\text {det }}$. In the case of deflagration, it is that of the shock driven ahead of the phase boundary, $v=v_{\mathrm{sh}}$. In both cases, $c_{s}<v<1$, where $c_{s}=1 / \sqrt{3}$ is the speed of sound. The expansion rate of the Universe can be ignored during this short period of rapid bubble growth. The fraction of space affected is then [2]

$$
F(t)=1-\exp \left[-\int_{t_{c}}^{t} d t^{\prime} p\left(t^{\prime}\right) \frac{4 \pi}{3} v^{3}\left(t-t^{\prime}\right)^{3}\right] .
$$

This grows rapidly from $F \simeq 0$ to $F \simeq 1$ at the phase transition time $t_{f}$, which we define by

$$
F\left(t_{f}\right) \equiv 1-1 / e .
$$

Bubble nucleation then ceases.

Let us note that for very slow deflagrations, $v_{\text {defl }} \lesssim 0.1$, the shock preceding the deflagration front is extremely weak. Between the shock and the deflagration front, the temperature increases continuously [3]. However, even the temperature at the deflagration front, $T_{q}$, may have been raised so little above the nucleation temperature, $T_{f}$, that the nucleation of new bubbles is not appreciably suppressed. Thus the velocity $v$ should be the deflagration velocity $v_{\text {deff }}$, instead of the shock velocity $v_{\text {sh. }}$. As will be seen below, this leads to much shorter distance scales.

Approximating

$$
S(t) \simeq S\left(t_{f}\right)-S^{\prime}\left(t_{f}\right)\left(t_{f}-t\right)
$$

and extending the integral to $-\infty$, Eq. 8 becomes

$$
S^{\prime}\left(t_{f}\right)^{4}=8 \pi v^{3} C T_{c}^{4} e^{-S\left(t_{f}\right)} .
$$

The typical distance $l_{n} \equiv n^{-1 / 3}$ between centers of neighboring bubbles is obtained from their number density

$$
n=\int d t^{\prime} p\left(t^{\prime}\right)\left[1-F\left(t^{\prime}\right)\right] \simeq C T_{c}^{4} \frac{e^{-S\left(t_{f}\right)}}{\left|S^{\prime}\left(t_{f}\right)\right|}=\frac{\left|S^{\prime}\left(t_{f}\right)\right|^{3}}{8 \pi v^{3}} .
$$

The above approximations require

$$
\frac{\left|S^{\prime \prime}\right|}{\left|S^{\prime}\right|^{2}} \ll 1
$$

and

$$
\left|S^{\prime}\right|\left(t_{f}-t_{c}\right) \gg 1
$$

From Eqs. 5 and 6 we obtain

$$
S\left(t_{f}\right)=\frac{A}{\hat{T}_{f} y^{2}},
$$




$$
S^{\prime}\left(t_{f}\right)=-\frac{A}{t_{c}} \frac{\hat{T}_{f}(8-9 y)}{2 y^{3}},
$$

and

$$
S^{\prime \prime}\left(t_{f}\right)=\frac{A}{t_{c}^{2}} \frac{\hat{T}_{f}^{3}}{4 y^{4}}\left(63 \hat{T}_{f}^{8}+34 \hat{T}_{f}^{4}-1\right),
$$

where

$$
A \equiv \frac{256 \pi}{3} \frac{\sigma^{3}}{L^{2} T_{c}}
$$

and

$$
y \equiv 1-\hat{T}_{f}^{4} .
$$

The nucleation time $t_{f}$ and temperature $T_{f}$ are solved from Eq. 10. Taking the logarithm and using Eqs. 14 and 6 it becomes

$$
y^{2}=\frac{A}{\hat{T}_{f} S\left(t_{f}\right)}=\frac{A}{\hat{T}_{f}\left[S_{A}-4 \ln \left|S^{\prime}\left(t_{f}\right) t_{c}\right|\right]},
$$

where $S_{A} \equiv \ln 8 \pi v^{3} C T_{c}^{4} t_{c}^{4} \simeq 4 \ln T_{c} t_{c} \simeq 170$. This gives an equation for $y$,

$$
y=\frac{A^{1 / 2}}{(1-y)^{1 / 8}\left[S_{A}-4 \ln \left|A(1-y)^{1 / 4}(8-9 y) /\left(2 y^{3}\right)\right|\right]^{1 / 2}},
$$

which can be solved iteratively. The phase transition temperature is then

$$
T_{f}=(1-y)^{1 / 4} T_{c}
$$

and the typical bubble distance

$$
l_{n}=\pi^{1 / 3} \frac{v}{A} \frac{2 y^{3}}{(1-y)^{1 / 4}(8-9 y)} t_{H},
$$

where $t_{H}=2 t_{c}$ is the Hubble distance at $t=t_{c}$. We plot these quantities in Fig. 1Conclusionsfigure.1. They depend on the parameters $\sigma$ and $L$ only through the combination 17 .

The approximations 12 and 13 fail near $\sigma^{3} / L^{2} T_{c}=0.25$ when the supercooling $1-\hat{T}_{f}$ and the distance scale $l_{n}$ are becoming very large. The nucleation rate, according to Eq. 5 , is beginning to grow more slowly and has a maximum at $T=T_{c} / \sqrt{3}$. This would indeed lead to extremely deep supercooling and very large distances. Of course, our equation of state 1, tuned to be valid near $T_{c}$, is then no longer applicable.

The critical radius

$$
r_{c}=\frac{2 \sigma}{p_{h}-p_{q}}=\frac{8 \sigma}{L y}
$$

must also not be too small $\left(\sim T_{c}^{-1}\right)$ for the above thin-wall nucleation to apply. From Fig. 2Conclusionsfigure.2 we see that for small $\sigma$, the critical radius is reasonably large, and almost independent of $L$. 
The values of $L$ and $\sigma$ indicated by pure glue lattice Monte Carlo simulations are


very large and, in particular, there is no lower limit. Thus, based on present knowledge, either one could be arbitrarily small. From Fig. 2Conclusionsfigure.2 we see that, e.g., length scales of $l_{n} / v t_{H} \approx 10^{-3}-10^{-2}$ are possible. This is a distance scale large enough to affect nucleosynthesis. The corresponding critical radii $r_{c}$ are large enough so that the thin-wall calculation should be valid.

\section{The effect of the equation of state}

Lattice Monte Carlo simulations imply that the energy density must have a very strong variation within a narrow temperature interval $(\leq 10 \mathrm{MeV})$ in QCD with physical quark masses [7]. Combined with the smallness of the latent heat this means that the realistic equation of state must differ even qualitatively from that of the bag model. This is illustrated in Fig. 3Conclusionsfigure.3. As has been discussed above, the parameter values of the naive bag model can be corrected to reproduce a desired latent heat. However, this corrected bag model ("bag model of text") cannot mimic at all the strong variation in the energy density. In this section we will analyze how the large derivative of the energy density curve, i.e., the large heat capacity, affects the nucleation.

In the case of the nucleation calculation we may assume that the Universe is, at least locally, in the quark phase and very near thermal equilibrium. Furthermore, we can ignore the tiny quark and lepton chemical potentials. We denote the cosmic scale factor of the Robertson-Walker metric by $R$, the energy density $\square$ by $\epsilon$, and the entropy density by $s$. From the equation $\mathrm{d}\left(\epsilon R^{3}\right)=-p \mathrm{~d}\left(R^{3}\right)$ it follows that

$$
\frac{\mathrm{d} R}{R}=-\frac{\mathrm{d} \epsilon}{3(\epsilon+p)}=-\frac{c(T)}{3 s(T)} \frac{\mathrm{d} T}{T},
$$

which can also be written as the equation $\mathrm{d}\left(s R^{3}\right)=0$ for entropy conservation. Here $c(T)$ is the density of heat capacity (specific heat) of the quark phase in constant volume. It satisfies the relations $c(T)=\mathrm{d} \epsilon / \mathrm{d} T=T(\mathrm{~d} s / \mathrm{d} T)=T\left(\mathrm{~d}^{2} p / \mathrm{d}^{2} T\right)$, where $p$ is the pressure. Equation 24 tells how the relation between expansion and cooling depends on the equation of state. Notice that in the case of the bag equation of state the factor $c / 3 s$ equals unity, and the simple relation $T \propto 1 / R$ is valid.

The expansion rate of the Universe is determined from the Friedmann equation,

$$
\frac{\dot{R}^{2}}{R^{2}}=\frac{8 \pi \epsilon(T)}{3 M_{\mathrm{Pl}}^{2}},
$$

\footnotetext{
${ }^{1}$ In this section, all quantities are measured in the supercooling quark phase.
} 
where $M_{\mathrm{Pl}}$ denotes the Planck mass. The vanishingly small curvature term was omitted. Eliminating $\mathrm{d} R / R$ from Eqs. 24 and 25 gives the cooling rate of the Universe:

$$
\mathrm{d} T=-\frac{3 s(T)}{c(T)} \times \sqrt{\frac{8 \pi \epsilon(T)}{3}} \frac{T}{M_{\mathrm{Pl}}} \mathrm{d} t .
$$

If the energy density curve $\epsilon(T)$ is very steep, the first factor is much smaller than unity. The Universe expands and the energy density decreases, but this causes only very slow cooling. In other words, the total energy density determines by Eq. 25 the expansion rate of the Universe, and this in turn determines by Eq. 24 the value of the derivative $\dot{\epsilon}(t)$; but then $\mathrm{d} T / \mathrm{d} t=\dot{\epsilon}(t) / c(T)$ is very small for large heat capacity.

Since the Universe cools slowly, it has time to nucleate even at a large action, and therefore the true nucleation temperature is slightly higher than that of the bag model. To see this, notice first that compared with the time spent in the supercooled state, the period of time during which essentially all the nucleation takes place is short, and the cooling rate 26 is practically constant during this period. We define the parameter $\delta$,

$$
\delta=\frac{c\left(T_{f}\right)}{3 s\left(T_{f}\right)},
$$

where $T_{f}$ is the temperature at the nucleation time $t_{f}$. In Fig. 3Conclusionsfigure.3, $\delta$ is roughly the ratio of slopes of the realistic equation of state, and the bag equation of state. The nucleation temperature is determined from Eq. 10. However, now the pressure difference $p_{h}(T)-p_{q}(T)$ is unknown, and thus is also the nucleation action $S(T)$. Still, we can find out what is the effect of $\delta$ : in the logarithm of Eq. 10 the value of the constant $S_{A} \simeq 170$ increases by $4 \log \delta$. As long as this increase is small compared with 170 the amount of supercooling, defined as $1-T_{f} / T_{c}$, decreases only little.

Although a large value of $\delta$ barely changes $T_{f}$, it has another significant effect. Physically the most important quantity related to nucleation is the average distance between nucleation centers immediately after the phase transition, $l_{n}$, defined between Eqs. 10 and 11. Its dependence on $\delta$ is seen from Eq. 11 to be

$$
l_{n} \propto \delta
$$

where the small correction coming from the change in $T_{f}$ was left out. This result tells that a steep drop in the energy density $\epsilon(T)$ increases significantly the typical distance between bubbles. This happens because when the cooling rate is lower it takes more time for the nucleation action $S(T)$ to decrease by a certain amount.

To get an order of magnitude estimate for $\delta$, we assume that the energy density changes from its asymptotic value in the quark phase to its asymptotic value in the hadron phase within $10 \mathrm{MeV}$. This is an upper limit indicated by lattice calculations [7]. Then we get $\delta \approx 8$. Thus the distance scales would be an order of magnitude larger than those shown in Fig. 2Conclusionsfigure.2. If the true temperature interval for the 
rapid change in energy density were smaller, say $1 \mathrm{MeV}$, then distance scales would be correspondingly larger, $\delta \approx 75$.

Finally, we note that the validity conditions of the nucleation calculation, Eqs. 12 and 13, do not depend in practice on $\delta$ unless the specific heat of the quark phase, $c(T)$, changes rapidly within the temperature interval $T_{c} \ldots T_{f}$.

\section{Detonations and deflagrations}

A bubble of $h$ phase surrounded by supercooled $q$ phase has two modes of growth available, detonations and deflagrations [8, 9, 10]. In a deflagration bubble the fluid inside the bubble is at rest, but the growing bubble is surrounded by a shock wave moving out ahead of the phase transition (deflagration) front. In a detonation bubble the phase transition (detonation) front advances into the fluid which is at rest, but is followed by a rarefaction wave where the fluid flows outwards following the detonation front. Relativistic detonation bubbles have been discussed in Ref. [11], and relativistic deflagration bubbles in Ref. [3].

Consider fluid flow through the phase transition front in the rest frame of the front. The inflow is subsonic for a deflagration, $v_{\text {in }}<c_{s}$, but supersonic for a detonation, $v_{\text {in }}>c_{s}$. These processes are further divided into weak and strong depending on the outflow velocity. For weak processes the nature of the flow velocity does not change, i.e., $v_{\text {out }}<c_{s}$ for weak deflagrations, and $v_{\text {out }}>c_{s}$ for weak detonations. For strong processes it changes, i.e., $v_{\text {out }}>c_{s}$ for strong deflagrations, and $v_{\text {out }}<c_{s}$ for strong detonations. The case where $v_{\text {out }}=c_{s}$ is called a Jouguet process.

In the rest frame of the unaffected fluid, all detonations and strong deflagrations move faster than sound, whereas weak deflagrations are subsonic.

Because of restrictions on the fluid flow pattern from the bubble geometry, strong detonations are not possible for phase transition bubbles [11]. (This is true also for 1-dimensional "bubbles").

In classical combustion theory for chemical burning the internal structure of the combustion front rules out strong deflagrations and weak detonations [8, 9]. In particular, the internal structure of a detonation front consists of a shock heating up the medium to initiate combustion, immediately followed by a deflagration. For a weak detonation, this deflagration would be a strong one. Thus the impossibility of strong deflagrations implies the impossibility of weak detonations.

The internal structure of a phase transition front is different from a combustion front. Heating by a shock does not facilitate the phase transition, and the structure of a detonation front is not shock+deflagration. Therefore weak detonations are not

ruled out [12]. Thus slowly growing bubbles $\left(v<c_{s}\right)$ will be weak deflagrations and fast ones $\left(v>c_{s}\right)$ will probably be weak detonations [13]. Strong deflagrations might also be possible in some cases. 
We denote by $T_{h}$ and $T_{q}$ the temperatures of the two phases at the phase transition front. For a detonation bubble, $T_{q}$ will be the phase transition temperature derived in Sec. 2Nucleationsection.2, $T_{q}=T_{f}$, but $T_{h}>T_{f}$, and we can even have $T_{h}>T_{c}$. The rarefaction wave cools the $h$ phase, so the final temperature will be below $T_{h}$ (and $T_{c}$ ). For a deflagration bubble, $T_{h}$ will be the final temperature of the $h$ phase, whereas $T_{q}$ is not the initial temperature $T_{f}$, but $T_{q}>T_{f}$ (and could exceed $T_{c}$ ), as the $q$ phase has been heated by the shock wave.

The detonation and deflagration solutions are obtained from the hydrodynamical conditions of energy and momentum conservation. These processes must also satisfy the condition of non-negative entropy production [8, 9, 10]. These constraints do not fix the process uniquely for a given initial temperature $T_{f}$. Instead, we have a one-dimensional family of allowed solutions for each $T_{f}$, with different temperatures $\left(T_{h}, T_{q}\right)$ and different bubble growth velocities $v_{\text {defl }}$ or $v_{\text {det }}$. This family may contain both deflagrations and detonations. Weak deflagrations are allowed for any $T_{f}<T_{c}$, but detonations and strong deflagrations require a minimum amount of supercooling, see Fig. 4Conclusionsfigure.4.

Below we give exact results for the bag equation of state 1 . If the energy densities are scaled by the bag constant $B=L / 4=\left(a_{q}-a_{h}\right) T_{c}^{4}$, and the temperatures by $T_{c}$, these results can be given in terms of a single parameter

$$
r \equiv \frac{a_{q}}{a_{h}}=\left[1-\frac{L}{4 a_{q} T_{c}^{4}}\right]^{-1}>1 .
$$

We identify a process by a point on the $\left(\epsilon_{h} / B, \epsilon_{q} / B\right)$-plane, where $\epsilon_{h}$ and $\epsilon_{q}$ are the energy densities of the two phases at the phase transition front. There are a number of special points in this plane (Fig. 4Conclusionsfigure.4).

Point C corresponds to $T_{h}=T_{q}=T_{c}$. This is the limit of weak deflagrations as $T_{f} \rightarrow T_{c}$. It is at the $v_{\text {defl }} \rightarrow 0$ limit (the diagonal line through $\mathrm{C}$ ). Point $\mathrm{D}$ is the (weak) detonation which requires the least supercooling. This point is at the $v_{\text {det }} \rightarrow 1$ limit (the diagonal line through $\mathrm{D}$ ). The velocities change steeply near these diagonals. Points $\mathrm{J}$ and $\mathrm{G}$ are the Jouguet detonations and deflagrations, respectively, requiring the least supercooling. The coordinates $\left(\epsilon_{h} / B, \epsilon_{q} / B\right)$ of these points are

$$
\begin{gathered}
C=\left(\frac{3}{r-1}, \frac{4 r-1}{r-1}\right), \\
D=\left(\frac{3 r}{r-1}, \frac{r+2}{r-1}\right), \\
J=\left(s_{J}+t_{J}, s_{J}-t_{J}\right), \\
G=\left(s_{G}+t_{G}, s_{G}-t_{G}\right),
\end{gathered}
$$

where

$$
s_{J}=\frac{2 r}{r-1}(1+\cos 2 \beta),
$$




$$
\begin{gathered}
t_{J}=2 \sqrt{\frac{2 r}{r-1}} \cos \beta \\
s_{G}=\frac{r}{r-1}\left(1+2 \sin ^{2} \beta+2 \sqrt{3} \cos \beta \sin \beta\right), \\
t_{G}=-\sqrt{\frac{2 r}{r-1}}(\cos \beta+\sqrt{3} \sin \beta),
\end{gathered}
$$

and

$$
\beta \equiv \frac{1}{3} \arctan \sqrt{\frac{r+1}{r-1}} .
$$

Converting the energy densities to temperatures by

$$
T_{h}^{4}=\frac{r-1}{3} \frac{\epsilon_{h}}{B} T_{c}^{4}, \quad T_{q}^{4}=\frac{r-1}{3 r} \frac{\epsilon_{q}-B}{B} T_{c}^{4},
$$

we have the following results. The maximum temperature for which the weak detonations are allowed is

$$
T_{f}=T_{q}(\mathrm{D})=r^{-1 / 4} T_{c} .
$$

At the detonation front we then have $T_{q}=r^{-1 / 4} T_{c}, T_{h}=r^{1 / 4} T_{c}$. The maximum temperature for which Jouguet detonations are allowed, is

$$
T_{f}=T_{q}(\mathrm{~J})=\left[\frac{r+1}{3 r}+\frac{2}{3} \cos 2 \beta-\frac{2}{3} \sqrt{2} \sqrt{\frac{r-1}{r}} \cos \beta\right]^{1 / 4} T_{c} .
$$

We always have $T_{q}(\mathrm{~J})<T_{q}(\mathrm{D})<T_{c}$.

For deflagration bubbles $T_{q} \neq T_{f}$. To relate these two temperatures we have numerically integrated the flow equations for the region between the shock and the deflagration fronts [3]. We denote the maximum temperature at which strong deflagrations are allowed by $T_{f}(\mathrm{G})$. The temperatures $T_{q}(\mathrm{D}), T_{q}(\mathrm{~J})$, and $T_{f}(\mathrm{G})$ are plotted as a function of $r$ in Fig. 5Conclusionsfigure.5.

If the Universe supercools very much, the phase transition is not able to reheat it back to $T_{c}$. The limiting supercooling temperature $T_{r}$ is obtained from

$$
\epsilon_{q}\left(T_{r}\right)=\epsilon_{h}\left(T_{c}\right)
$$

For the bag equation of state 1, this gives

$$
T_{r}=\left(\frac{4-r}{3 r}\right)^{1 / 4} T_{c}=\left(\frac{1}{r}-\frac{r-1}{3 r}\right)^{1 / 4} T_{c} .
$$

If $T_{f}>T_{r}$, the phase transition reheats the Universe to $T_{c}$ before completing. The rapid detonation/deflagration stage is followed by a slower stage, where both phases coexist in thermal equilibrium at $T_{c}$, and the phase transition proceeds only as the Universe expands [14]. If $T_{f}<T_{r}$, the detonation/deflagration takes the transition to 
completion. If $r>4$, i.e., $B>\epsilon_{h}\left(T_{c}\right)$, then reheating to $T_{c}$ is possible from arbitrarily low temperatures.

Comparing Eq. 40 with Eq. 43 we note that $T_{r}$ is always below $T_{q}(\mathrm{D})$, although for small $r$, these are close to each other. Thus in those cases where the Universe has supercooled so much that it will not reheat back to $T_{c}$, weak detonations are always allowed. The similar statement for Jouguet detonations becomes true for $r>1.644$.

In Sec. 2Nucleationsection.2 we related $T_{f}$ to $\sigma$ and $L$. The parameter $r$ depends on $a_{q}$ in addition to $L$. By making some assumption about $a_{q}$, we can convert $\sigma$ and $L$ to $T_{f}$ and $r$, and classify points in the $(L, \sigma)$ parameter space according to which processes are allowed. This is done in Fig. 6Conclusionsfigure.6.

As a concrete example, let us inspect the case $\sigma=0.01$. Now it is seen from Fig. 6Conclusionsfigure.6 that if $L=1$, only weak deflagrations are allowed and the Universe does not reheat to the critical temperature. If $L=0.1$, weak deflagrations are possible as well, and the Universe reheats back to $T_{c}$. If $L=0.01$ even Jouguet processes and strong deflagrations are allowed.

To summarize the results of this section, for a given phase transition temperature $T_{f}<T_{c}$, there is a one-dimensional family of allowed bubble growth processes. This family will always include weak deflagrations. It may also include weak detonations (if $T_{f}<T_{q}(\mathrm{D})$ ), Jouguet detonations (if $T_{f} \leq T_{q}(\mathrm{~J})$ ), Jouguet deflagrations (if $T_{f} \leq$ $T_{f}(\mathrm{G})$ ), and strong deflagrations (if $T_{f}<T_{f}(\mathrm{G})$ ). Which of these allowed processes actually occurs, depends on the dissipative mechanisms internal to the front which determine the propagation speed of the transition [8, 9, 13]. Even though external conditions may allow detonations, the actual process could still be a slowly propagating weak deflagration.

\section{Baryon number}

Much of the interest in the QCD phase transition in cosmology stems from the possibility of leaving behind strong inhomogeneities in the baryon number, and maybe thus affecting big bang nucleosynthesis. The baryon number in the $q$ phase is carried by massless quarks, but in the $h$ phase it is carried by nucleons, with $m \gg T_{c}$. The baryon number does not penetrate the phase boundary easily, and accumulates as a layer on the $q$ side of the phase boundary. As more baryon number accumulates onto this layer, more will also leak through, but the net effect is that of dragging baryons towards the regions which remained longest in the $q$ phase [15, 16, 17, 18].

Even assuming we know the hydrodynamic details of the phase transition discussed above, it is difficult to estimate the shape and density contrast of the baryon number inhomogeneity. It depends on the rate of baryon transport within each phase and baryon penetration of the boundary, which are not known.

To have a significant effect on nucleosynthesis, a number of conditions need to 
be satisfied. 1) The distance scale should be large, $l_{n}>1 \mathrm{~m} \approx 10^{-4} t_{H}$. 2) The high-density regions should contain most of the total baryon number. 3) The density contrast $R$, i.e., the ratio of baryon number density in the high-density region to that in the low-density region, must be large, $R_{\text {final }}>\left(n_{p} / n_{n}\right)_{\text {nucleosynthesis }} \sim 7$.

We have argued in Sections 2Nucleationsection.2 and 3The effect of the equation of statesection. 3 that condition 1 could be satisfied. Because of the difficulty of getting baryon number through the phase boundary, condition 3 does not appear unreasonable. Condition 2 is perhaps the most difficult [16].

During the phase transition, baryon number has been collected onto a layer on the surface of the bubble, with some thickness $d$. The baryon density in this layer is $R_{\text {layer }}$ times larger than elsewhere. To have most of the baryon number in this layer, we must have roughly $R_{\text {layer }}>l_{n} / d$. If the thickness of the layer is due to microscopic diffusion of baryon number away from the boundary (in the $q$ phase), $d$ will be very small. It has been argued that turbulent transport will be much more effective than microscopic diffusion, and lead to a much thicker layer, so that condition 2 might be satisfied 19

In the usual picture of this phase transition, most of the growth of the $h$ regions will happen in the equilibrium stage of the transition, very slowly. In the picture we have presented here, which leads to large distance scales $l_{n}$ even with a small surface tension $\sigma$, this stage does not usually exist. The phase transition is completed by detonation or deflagration.

Especially, if the bubble growth process is a detonation, the phase transition is completed rapidly. The detonation bubbles grow with $v_{\text {det }}>c_{s}$. In those regions where the detonation bubbles collide, the transition is then already completed, and the turbulence caused by the collision will operate only in the $h$ phase. Where there is any $q$ phase left, the detonation front will move on unaffected, and once they have covered the space between them, the transition is over. The baryon number accumulating on the phase boundary cannot escape from the supersonically moving front. Thus the layer should get no thicker than a few fm. This appears to make condition 2 impossible to satisfy.

If the bubble growth process is a deflagration, the phase boundary will be slower and turbulent baryon transport may be effective in making the layers thicker. As mentioned in Sec. 2Nucleationsection.2, the distances between nucleated bubbles do not depend on whether the bubbles grow as deflagrations or detonations, unless the deflagrations are exceedingly slow. Therefore, slowly growing deflagration bubbles, with efficient turbulent baryon transport, seem to be able to affect nucleosynthesis.

If the hadron bubbles grow as deflagrations, but with an exceedingly low velocity, there may be another mechanism, in addition to turbulence, for enhancing the baryon number. Neutrinos, which do not carry any baryon number, may carry a considerable part of the energy flux. The reason is that the hydrodynamical flux $w \gamma^{2} v$, measured 
in the rest frame of the phase transition front, vanishes as $v \rightarrow 0$. This curve is given as the solution of the equation $p_{q}\left(T_{q}\right)=p_{h}\left(T_{h}\right)$. The solution does not agree with the curve $T_{q}=T_{h}$, so the neutrino flux $\left(g_{* n} \pi^{2} / 120\right)\left(T_{q}^{4}-T_{h}^{4}\right)$, where $g_{* n}$ denotes the effective number of active neutrino degrees of freedom, remains non-zero. But as noted above, for these very slow deflagrations the distance scales are also small. However, according to Sec. 3The effect of the equation of statesection.3 an extremely large heat capacity would increase the distance scale with several orders of magnitude. There is also another escape: suppose that the initial growth mechanism is a deflagration which is not very slow, so that distance scales are large, but that then the Universe reheats to $T_{c}$, where a stage of very slow growth near thermodynamical equilibrium follows. Here, neutrino transport may be effective [20], in addition to turbulence. If this stage lasts long enough, the baryon number remaining at the high-density regions could be enhanced. From Figs. 2Conclusionsfigure.2 and 6Conclusionsfigure.6 one can see that this scenario is possible in a very small but non-vanishing region of the parameter space, near e.g. the point $L \approx 2 T_{c}^{4}, \sigma \approx 0.3 T_{c}^{3}$.

Finally, let us note that so far we have only studied which hydrodynamical processes are in principle possible, without being able to fix a definite growth velocity. In Ref. [13] a model is presented which tries to fix this velocity, by introducing a phenomenological dissipative constant $\Gamma$. With a dimensional estimate $\Gamma \approx 1 T_{c}^{-1}$, and the parameters $L \approx 0.1 T_{c}^{4}, \sigma \approx 0.1 T_{c}^{3}$, the bubbles grow as deflagrations, with a velocity $v_{\text {defl }}=0.1$. The neutrino flux is vanishingly small, but turbulence might be effective.

\section{Conclusions}

Parametrizing the QCD phase transition with the latent heat $L$ and the surface tension $\sigma$, we have studied bubble nucleation and growth. The effects of a more general parametrization have been estimated. We have investigated the possibility that the inhomogeneities generated at the QCD phase transition significantly affect nucleosynthesis.

We find that parameter values in the range of, e.g., $\sigma \approx 0.01-0.1 T_{c}^{3}, L \approx 0.01-$ $0.1 T_{c}^{4}$, lead to a transition with large supercooling, relatively large critical bubbles, and a distance between bubbles of $l_{n} \approx 10^{-3}-10^{-2} t_{H}$. These bubbles may grow as detonations as well as deflagrations, and the Universe does not reheat to $T_{c}$. In spite of the large distance scale of the inhomogeneity, phase we found that for detonations the accumulation of baryon number in the high density regions is too ineffective to make a noticeable effect on nucleosynthesis likely. Even in the new region of parameter space studied, only deflagrations with efficient turbulent baryon number transport, or maybe with large neutrino flux, seem able to affect nucleosynthesis. 


\section{References}

[1] J. H. Applegate, C. J. Hogan, and R. J. Scherrer, Phys. Rev. D 35, 1151 (1987).

[2] A. Guth and E. Weinberg, Phys. Rev. D 23, 876 (1981).

[3] H. Kurki-Suonio, Nucl. Phys. B 255, 231 (1985).

[4] Y. Iwasaki et al. (QCDPAX Collaboration), Phys. Rev. D 46, 4657 (1992).

[5] B. Grossmann and M. L. Laursen, Nucl. Phys. B 408, 637 (1993).

[6] Y. Iwasaki, K. Kanaya, L. Kärkkäinen, K. Rummukainen, and T. Yoshié, Phys. Rev. D 49, 3540 (1994).

[7] B. Petersson, Nucl. Phys. B (Proc. Suppl.) 30, 66 (1993).

[8] L. D. Landau and E. M. Lifshitz, Fluid Mechanics, 2nd edition (Pergamon Press, Oxford, 1987).

[9] R. Courant and K. O. Friedrichs, Supersonic flow and shock waves (Springer-Verlag, Berlin, 1985).

[10] M. Gyulassy, K. Kajantie, H. Kurki-Suonio, and L. McLerran, Nucl. Phys. B 237, 477 (1984).

[11] P. J. Steinhardt, Phys. Rev. D 25, 2074 (1982).

[12] M. Laine, Phys. Rev. D, in press.

[13] J. Ignatius, K. Kajantie, H. Kurki-Suonio, and M. Laine, Phys. Rev. D, in press.

[14] K. Kajantie and H. Kurki-Suonio, Phys. Rev. D 34, 1719 (1986).

[15] G. M. Fuller, G. J. Mathews, and C. R. Alcock, Phys. Rev. D 37, 1380 (1988).

[16] H. Kurki-Suonio, Phys. Rev. D 37, 2104 (1988).

[17] J. I. Kapusta and K. A. Olive, Phys. Lett. B 209, 295 (1988).

[18] K. Sumiyoshi, T. Kajino, C. R. Alcock, and G. J. Mathews, Phys. Rev. D 42, 3963 (1990).

[19] G. J. Mathews, B. S. Meyer, C. R. Alcock, and G. M. Fuller, Astrophys. J. 358, 36 (1990).

[20] J. H. Applegate and C. J. Hogan, Phys. Rev. D 31, 3037 (1985). 


\section{FIGURE CAPTIONS}

Fig. 1: The supercooling $1-\hat{T}_{f}$ and the bubble distance scale $l_{n}$. The distance scale is given as $l_{n} / v t_{H}$, where $t_{H}$ is the Hubble distance ("horizon") and $v$ is the detonation or shock velocity, $1 / \sqrt{3}<v<1$. The dependence on the surface tension $\sigma$ and the latent heat $L$ is through the combination $\sigma^{3} / L^{2} T_{c}$. We also show the nucleation action $S\left(t_{f}\right)$. The other thin lines show the two quantities $S^{\prime \prime}\left(t_{f}\right) / S^{\prime}\left(t_{f}\right)^{2}$ (short-dashed line) and $\left[\left|S^{\prime}\left(t_{f}\right)\right|\left(t_{f}-t_{c}\right)\right]^{-1}$ (dotted line), whose smallness we have assumed. Our approximations are seen to break down at $\sigma^{3} / L^{2} T_{c} \gtrsim 0.25$.

Fig. 2: Contours of the critical radius $r_{c}$ and the bubble separation $l_{n}$ on the $(L, \sigma)$ parameter plane. The solid line corresponds to $\sigma^{3} / L^{2} T_{c}=0.25$. This figure is for the bag equation of state. equation of As discussed in the text, use of a more realistic equation of state could increase the distances $l_{n}$ by an order of magnitude or more.

Fig. 3: Schematic representation of the energy density versus $T^{4}$ for three different equations of state. Thinner parts of the curves denote the metastable branches. For clarity the magnitude of $L$ has been exaggerated in the figure. 
Fig. 4: Detonations and deflagrations: This plot shows how the different processes lie in the $\left(\epsilon_{h}, \epsilon_{q}\right)$-plane. The entropy condition restricts the allowed processes below the $\Delta S=0$ curve. Point $\mathrm{C}$ corresponds to $T_{q}=T_{h}=T_{c}$. For a given $T_{f}$ there is a 1dimensional family of solutions, denoted by the dashed line. The detonation branch of this family is a horizontal line, the deflagration branch a steep curve. For any $T_{f}<T_{c}$ it always passes to the left of point $\mathrm{C}$, indicating that weak deflagrations are allowed. If it passes to the left of $\mathrm{G}$, strong deflagrations are allowed. If the detonation branch passes below D $(\mathrm{J})$, then weak (Jouguet) detonations are allowed. This figure is for $r=1.01$.

Fig. 5: Some special temperatures for the bag equation of state as a function of $r$. $T_{q}(\mathrm{D})$ is the maximum temperature for which (weak) detonations are allowed. $T_{q}(\mathrm{~J})$ is the maximum temperature for which Jouguet detonations are allowed. $T_{f}(\mathrm{G})$ is the maximum temperature for which Jouguet deflagrations are allowed. For $T<T_{f}(\mathrm{G})$, strong deflagrations are allowed. $T_{r}$ is the lowest temperature for which the latent heat is sufficient to reheat the universe back to $T_{c}$.

Fig. 6: Regions on the $(L, \sigma)$ parameter space, where different processes are allowed. This figure is for a bag model with $a_{q}=34.25 \pi^{2} / 90+L / 8 T_{c}^{4}, a_{h}=34.25 \pi^{2} / 90-L / 8 T_{c}^{4}$. The solid lines divide the graph in three regions depending on what kind of detonation bubbles are allowed by hydrodynamic considerations. Weak deflagrations are always allowed. Strong deflagrations are allowed above the long-dashed line. The universe reheats to $T_{c}$ if we are below the short-dashed line. Thus detonations are always allowed in those cases where the universe does not reheat to $T_{c}$. 
This figure "fig1-1.png" is available in "png" format from: http://arxiv.org/ps/hep-ph/9405336v1 
This figure "fig1-2.png" is available in "png" format from: http://arxiv.org/ps/hep-ph/9405336v1 
This figure "fig1-3.png" is available in "png" format from: http://arxiv.org/ps/hep-ph/9405336v1 
This figure "fig1-4.png" is available in "png" format from: http://arxiv.org/ps/hep-ph/9405336v1 
This figure "fig1-5.png" is available in "png" format from: http://arxiv.org/ps/hep-ph/9405336v1 
This figure "fig1-6.png" is available in "png" format from: http://arxiv.org/ps/hep-ph/9405336v1 